\title{
Late main-sequence evolution of lithium and beryllium
}

\author{
L. Piau ${ }^{1}$, S. Randich ${ }^{2}$, and F. Palla ${ }^{2}$ \\ 1 Institut d'Astronomie et d'Astrophysique, ULB, CP226, 1050 Brussels, Belgium \\ e-mail: piau@astro.ulb.ac.be \\ 2 INAF-Osservatorio Astrofisico di Arcetri, Largo E. Fermi 5, 50125 Firenze, Italy \\ e-mail: randich@arcetri.astro.it;palla@arcetri.astro.it
}

Received 26 December 2002 / Accepted 4 June 2003

\begin{abstract}
We examine the effects of the tachocline diffusion process on the surface abundances of light elements in solarlike stars $\left(M_{*}=0.96-1.04 M_{\odot}\right)$. Acting during main sequence evolution, the tachocline diffusion can account for the gradual decrease in lithium surface abundance while preserving beryllium, in agreement with the most recent observational data for open cluster stars older than the Hyades ( $\sim 600 \mathrm{Myr}$ ). We show that helioseismology and observations of surface rotation demand a nearly solid-body rotation of solar analogs after $\approx 1 \mathrm{Gyr}$. By then, these stars have become slow rotators and lost most of their initial angular momentum. We argue that mixing due to angular momentum loss does not appear to be a viable mechanism to account for the observed abundances.

Using the results of helioseismology and of the observations of solar lithium abundance, we calibrate the two parameters of the tachocline mixing, the Brunt-Väisälä frequency and the tachocline thickness. We then evolve stellar models starting at the Hyades age and compare the results of ${ }^{7} \mathrm{Li}$ and ${ }^{9} \mathrm{Be}$ abundances to the observations of M 67 ( 4.5 Gyr). Finally, we investigate the effects of slight differences in specific metal abundance ratios on the tachocline diffusion. Because of opacity effects, we find differences in the amount of lithium depletion of $\sim 0.5$ dex for solar effective temperature and iron abundance at the age of M 67 that can partly explain the observed lithium spread in this cluster.
\end{abstract}

Key words. stars: evolution - stars: rotation - stars: interiors - stars: abundances

\section{Introduction}

The light elements lithium, beryllium, and boron $(\mathrm{LiBeB})$ are very powerful tracers of stellar structure and stellar interiors, due to their low, but progressively higher, burning temperatures. In particular, ${ }^{7} \mathrm{Li}$ and ${ }^{9} \mathrm{Be}$ are destroyed through proton capture in stellar plasma conditions exceeding temperatures of $\sim 2.5 \times 10^{6} \mathrm{~K}$ and $\sim 3.5 \times 10^{6} \mathrm{~K}$, respectively. Thus, these elements are depleted from stellar surfaces as soon as a mixing mechanism operates that is able to transport material deep enough in the stellar interior at these characteristic temperatures. Their abundance evolution hence probes the extent of outer convection zones and the role of possible additional mixing processes.

A large number of surveys of $\mathrm{Li}$ abundances in open cluster stars have been carried out in the last decade with the aim of setting empirical constraints on the nature of mixing processes responsible for its depletion (e.g., Jeffries 2000; Pasquini 2000 and references therein). As a result, it has become possible to trace the evolution of lithium from the age of the youngest clusters up to a few Gyr. The distribution of $\mathrm{Li}$ abundances of young clusters (35-50 Myr) shows that solar-type stars undergo very little (if any) Li depletion during the pre-main sequence (pre-MS) phases (e.g., Martìn \& Montes 1997; Randich et al. 2001). On the contrary, these stars do deplete $\mathrm{Li}$ on the main sequence (MS), possibly during two main phases. In the first one, that lasts approximately from the zero age main sequence (ZAMS) to the age of the Hyades (600 Myr), a factor of $\sim 3-5 \mathrm{Li}$ depletion occurs in a characteristic time of $\approx 500 \mathrm{Myr}$. In the second episode, a similar (or even smaller) amount of lithium is destroyed on a much longer timescale of at least a few Gyr, as evidenced by the observations of the $\sim 2$ Gyr clusters IC 4651 and NGC 3680 (Randich et al. 2000), of M 67 ( 4.5 Gyr, Jones et al. 1999), and of the $\sim 6$ Gyr old cluster NGC 188 (Randich et al. 2003). Solar-type stars in all these clusters have very similar maximum $\mathrm{Li}$ abundances which are only a factor of 2-3 below the Hyades. The efficiency of this second phase can vary strongly from star to star and from cluster to cluster. In M 67 and in the field, stars with an additional depletion as large as a factor of 10 to 30 are observed, resulting in a large $\log n(\mathrm{Li})$ dispersion (Jones et al. 1999; Pasquini et al. 1994). This suggests that the depletion history depends on at least one additional parameter besides mass and age. We mention that the Sun with a lithium abundance $\log n(\mathrm{Li})=1.16$ (Anders \& Grevesse 1989), i.e. a factor $\sim 100$ below the initial meteoritic value, belongs to the group of Li-poor stars.

With the exception of pioneering studies of beryllium in the Hyades (e.g., Boesgaard \& Budge 1989; García López et al. 1995), observational efforts have mostly concentrated on lithium and only very recently beryllium has been measured in a relatively large sample of cluster stars (in particular in old 
clusters), thanks to the high near-UV efficiencies of the HIRES spectrograph on the Keck telescope and of the UVES spectrograph on the VLT UT2 (Boesgaard \& King 2002; Randich et al. 2002). These observations have shown that at variance with ${ }^{7} \mathrm{Li},{ }^{9} \mathrm{Be}$ is not depleted in solar-type stars (including the Sun) during MS evolution up to at least the solar age.

The theoretical framework of lithium depletion is quite complex. Standard models fail to reproduce the observational scenario. In particular, they do not predict any Li-depletion during the MS phase of solar-type stars since the outer convection zones are too shallow. A number of non-standard physical processes have been proposed to explain the observed depletion: diffusion (Chaboyer et al. 1995), mass loss (Swenson \& Faulkner 1992), slow-mixing processes driven by gravity waves and/or by rotational instabilities (Montalbán \& Schatzmann 2000; Deliyannis \& Pinsonneault 1997). On the other hand the evolution of beryllium in G-type stars has been less investigated theoretically than lithium because of the lack of observational constraints. Nevertheless, each of the previous mechanisms makes very precise predictions on Be abundances and on the Be/Li ratios. As summarized by Deliyannis (2000), diffusion implies simultaneous $\mathrm{Li}$ and $\mathrm{Be}$ depletion for stars cooler than $\sim 6600 \mathrm{~K}$; models including mass loss would predict a flat Be vs. Li distribution, since all lithium must have disappeared before Be destruction starts; finally, according to models including slow (angular momentum loss induced) mixing, lithium should be depleted more efficiently than beryllium, but a Be vs. Li correlation is predicted.

None of the above non-standard processes seems able to fully explain the observed abundance patterns. In fact, the microscopic diffusion time is much longer than the characteristic depletion duration of $\approx 500 \mathrm{Myr}$ on the early MS and of $\approx 1-2$ Gyr past the Hyades age. In addition, models including diffusion do not fit the observed Be vs. Li diagram of old cluster stars (Randich et al. 2002). Also, mass loss is not consistent with the $T_{\text {eff }}-\log n(\mathrm{Li})$ pattern in the Hyades cluster (e.g. Swenson \& Faulkner 1992) and would require anomalously high mass-loss rates. Fast processes, such as overshooting, are easily excluded as the observed characteristic depletion time is larger than a few $100 \mathrm{Myr}$, while overshooting is a very fast process. Finally, current models including mixing driven by angular momentum loss are unable to reproduce the observed $\mathrm{Li}$ and Be patterns in old clusters, in particular the Be vs. Li diagram (Randich et al. 2002). We stress here that the absence of Be depletion in stars with temperatures in the interval 5400 to $6000 \mathrm{~K}$ suggests that the additional mixing responsible for the slow disappearance of ${ }^{7} \mathrm{Li}$ affects only a narrow region at the upper convection zone. The situation is less clear around the lithium dip temperature (e.g. Balachandran 1995 and references therein) where both lithium and beryllium appear to be depleted (Boesgaard \& King 2002). Similarly, K-type stars may also experience ${ }^{9} \mathrm{Be}$-depletion due to the deep extent of the convection zone. However, the study of these type of stars is beyond the scope of the present work that focuses on solartype objects.

In this paper, we address the problem of the evolution of the surface abundance of lithium and beryllium during the middle and late phases of the main sequence (MS) of solar-type stars using the CESAM (Morel 1997) evolution code in the mass range $0.95 M_{\odot}<M_{*}<1.05 M_{\odot}$. For solar composition, this mass interval corresponds to effective temperatures [5500-5900 K] at the Hyades age and [5600-6000 K] at the solar age. More specifically, we study the tachocline mixing or diffusion (Spiegel \& Zahn 1992) as a possible depletion mechanism operating from the age of the Hyades to that of M 67 and the Sun. This process is a hydrodynamic process and is not related to magnetic effects. It is induced by a time dependent rotation and by differential rotation with latitude and it has been successfully introduced in solar modeling by Brun et al. (1999, hereafter BTZ). The tachocline mixing brings two major improvements to solar/stellar models: first, it decreases the discrepancy between the observed and the theoretical sound speed near the top of the solar radiative zone; second, it implies a slow decrease of the surface lithium abundance with a characteristic time scale of $\sim 1$ Gyr for MS solar analogs. As we will show, this mechanism does not affect the beryllium fraction and thus appears as a good candidate to explain Li and Be evolution in open cluster solar-type members.

In the following section, we describe the tachocline mixing and our prescriptions for the rotational evolution of solar mass stars. Based on observations, we set constraints on the internal rotational properties and on the related mixing processes. In Sect. 3 we discuss empirical constraints on the parameters driving the tachocline mixing, extending the work of BTZ to masses slightly larger/smaller than solar. Variations of the metal abundances in the gas opacity are discussed in Sect. 4, along with the effects on the spread of lithium depletion. The main results are summarized in the last section.

\section{The model}

\subsection{Tachocline mixing}

We briefly summarize here the prescriptions for tachocline mixing following Spiegel \& Zahn (1992). In order to solve the hydrodynamical equations describing the tachocline region, Spiegel \& Zahn project the variables on horizontal eigenfunctions $F_{i}$ and express the diffusive coefficient efficiency as a function of depth by:

$D_{t}(r)=\Sigma_{i} A_{i} K\left(2 \Omega / N_{\mathrm{BV}}\right)^{2}\left(r_{\mathrm{bzc}} / d_{\mathrm{tacho}}\right)^{2} \mathrm{e}^{-2 \zeta_{i}} \cos ^{2} \zeta_{i}$,

where $K$ and $\Omega$ are the radiative diffusivity and angular speed at the base of the convection zone, respectively. $r_{\mathrm{bzc}}$ is the lower radius of the convective region and $\zeta_{i}=\mu_{i}\left(r_{\mathrm{bzc}}-r\right) / d_{\mathrm{tacho}}$, where $\mu_{i}$ is the eigenvalue associated with the eigenfunction $F_{i}$. The coefficient $A_{i}$ depends on the differential rotation rate with latitude, and decreases rapidly with increasing index $i$. In Eq. (1), $N_{\mathrm{BV}}$ is the Brunt-Väisälä (or buoyancy) frequency, and $d_{\text {tacho }}$ is twice the tachocline thickness $h_{\text {tacho. These two }}$ parameters play quite distinct and important roles. On the one hand, $N_{\mathrm{BV}}$ dictates the absolute values of the turbulent diffusivity: small values of $N_{\mathrm{BV}}$ imply weaker restoring forces to vertical perturbations and therefore favor turbulent mixing. The limiting case is $N_{\mathrm{BV}}=0 \mu \mathrm{Hz}$, by then convection sets in. On the other hand, $d_{\text {tacho }}$ regulates the slope of the decrease of turbulent diffusivity below the convection zone and represents the 
typical extent of the mixing layer. Quantitatively, both effects translate in the formulation of $D_{\mathrm{t}}$, whose absolute value is proportional to $N_{\mathrm{BV}}^{-2}$, and whose exponential decrease with depth scales as $1 / d_{\text {tacho }}$. Therefore, both $N_{\mathrm{BV}}$ and $d_{\text {tacho }}$ affect the extent of the mixing region, but in different ways.

The numerical values of these two free parameters must be estimated using empirical constraints. $N_{\mathrm{BV}}$ is rather problematic as it is a very steep function of depth near the radiation/convection transition. In the present Sun, we have $N_{\mathrm{BV}}=$ $0.11 \mu \mathrm{Hz}$ at $R=0.71 R_{\odot}$, and $N_{\mathrm{BV}}=153 \mu \mathrm{Hz}$ at $R=0.69 R_{\odot}$, close to the typical values of the radiative interior where $N_{\mathrm{BV}}=$ 200 to $400 \mu \mathrm{Hz}$. The tachocline thickness can be inferred from the observed width of the transition region from the differentially rotating convective zone to the radiative region in solid body rotation. Helioseismic observations indicate a tachocline thickness $h_{\text {tacho }}$ in the range 0.01 to $0.05 R_{\odot}$ (Corbard et al. 1999; Corbard et al. 2000; Charbonneau et al. 1999). On the other hand, MHD calculations of the dynamo generated solar magnetic field require $h_{\text {tacho }}>0.05 R_{\odot}$ in order to reproduce the solar cycle and not to have too many belts of toroidal field (Rüdiger \& Brandenburg 1995). In the following, we will assume $h_{\text {tacho }}=0.025 R_{\odot}$. This value represents the mean of various estimates based on helioseismological grounds and is close to the most probable value of $0.019 R_{\odot}$ suggested by Elliott $\&$ Gough (1999). Models with larger values of the tachocline will also be considered to account for the full range of possible values. In Sect. 3, we will constrain possible values of the tachocline parameters using both the recent observations of $\mathrm{Li}$ and $\mathrm{Be}$ and helioseismology. Before that, we must discuss the stellar rotational history of the stellar models which represents a key ingredient for the tachocline mixing.

\subsection{Rotational evolution}

We consider the structural and angular momentum evolution of stars with masses $0.96,0.98,1.00$, and $1.04 M_{\odot}$ between the birth-line and $\sim 6$ Gyr. The following assumptions are made: i) The convective envelope and the radiative core rotate as solid bodies with possible different angular speeds; ii) The star initially rotates as a solid body with an initial period $P_{\mathrm{o}}=8$ days. This choice is motivated by the convective state of the stars at the beginning of their pre-MS evolution and by the rotational rates observed among classical T Tauri stars (e.g., Attridge \& Herbst 1992; Choi \& Herbst 1996). We have also assumed a lifetime of the initial circumstellar disk of $\tau_{\mathrm{d}}=0.5 \mathrm{Myr}$ which implies a very fast rotation and angular momentum on the ZAMS (e.g., Bouvier et al. 1997). Initial rotation periods of $\mathrm{T}$ Tauri stars exhibit a bimodal distribution for $1 M_{\odot}$ stars and range in the interval $\sim 2-16$ days (Herbst et al. 2000). This is however of no incidence for the present study because stars achieve similar rotation rates after a few $100 \mathrm{Myr}$ whatever the initial conditions: we compute that models with $P_{\mathrm{o}}=4$ days, $\tau_{\mathrm{d}}=0.3 \mathrm{Myr}$ and $P_{\mathrm{o}}=8$ days, $\tau_{\mathrm{d}}=10 \mathrm{Myr}$ reach 8.9 and 10.4 days rotational period at Hyades age, respectively. This difference gets subsequently smaller; iii) The internal structural changes are taken into account all along the evolution. However, their impact on rotation mainly concerns the pre-MS contraction when the radiative core develops rapidly, i.e. in the initial 230 Myr. Prior to this phase, rotation is too slow to produce significant angular momentum loss (see below). As the angular momentum $J=I \Omega$ remains constant and the moment of inertia $I$ decreases, the star spins-up quickly and the equatorial surface velocity peaks to a maximum on the ZAMS ( $50 \mathrm{Myr})$; iv) The angular momentum loss is described using Kawaler (1988) prescription:

$$
\begin{aligned}
& \frac{\mathrm{d} J}{\mathrm{~d} t}=-K \Omega^{3}\left(\frac{R}{R_{\odot}}\right)^{1 / 2}\left(\frac{M}{M_{\odot}}\right)^{-1 / 2}, \Omega<\Omega_{\text {sat }} \\
& \frac{\mathrm{d} J}{\mathrm{~d} t}=-K \Omega \Omega_{\text {sat }} 2\left(\frac{R}{R_{\odot}}\right)^{1 / 2}\left(\frac{M}{M_{\odot}}\right)^{-1 / 2}, \Omega>\Omega_{\text {sat }} .
\end{aligned}
$$

For the solar mass model, we have taken $K=3.25 \times$ $10^{47} \mathrm{~g} \mathrm{~s} \mathrm{~cm}^{2}$, and $\Omega_{\text {sat }}=14 \Omega_{\odot}$ (see discussion in Piau $\&$ Turck-Chièze 2002). In agreement with the observed relation between $\Omega_{\text {sat }}$ and mass (e.g., Krishnamurthi et al. 1997; Bouvier et al. 1997), we assume a linear scaling so that $\Omega_{\text {sat }}=$ $\left.\left[14+\left(M_{\star}-M_{\odot}\right) \times 6 / 0.2\right] \times \Omega_{\odot} ; v\right)$ If the core and the envelope exchange angular momentum by an amount

$$
\Delta J=\frac{\left(I_{\text {env }} J_{\text {core }}-I_{\text {core }} J_{\text {env }}\right)}{I_{\text {core }}+I_{\text {env }}},
$$

they would achieve the same angular speed. Following MacGregor \& Brenner (1991), we consider that this factor is modulated by a characteristic coupling time $\tau_{\mathrm{c}}$ so that after a time interval $\mathrm{d} t$ the transfer of angular momentum is $\mathrm{d} J=\frac{\Delta J}{\tau_{\mathrm{c}}} \mathrm{d} t$. Many processes contribute to establishing the coupling between core and envelope. They could be of hydrodynamical or hydromagnetical nature (see Pinsonneault et al. 1989 and Barnes et al. 1999). They could also be related to internal gravity waves (Zahn et al. 1997; Talon et al. 2002). However, whatever the physical nature of the coupling, $\tau_{\mathrm{c}}$ is not entirely a free parameter, since both helioseismology and the observation of surface rotation rates limit its possible values, as we will show below.

\subsection{Differential rotation with depth}

In Fig. 1, we plot the evolution of the ratio of the angular velocity between the radiative and convective zones $\Omega_{\text {core }} / \Omega_{\text {env }}$ for solar models with values of $\tau_{\mathrm{c}}$ equal to 50,100, 200, and $300 \mathrm{Myr}$. The figure shows that for $\tau_{\mathrm{c}}=300 \mathrm{Myr}, \Omega_{\text {core }} / \Omega_{\mathrm{env}} \approx$ 4 at $4.5 \mathrm{Gyr}$; such a large differential rotation is excluded by current helioseismic observations (Corbard et al. 1997; Schou et al. 2002), implying that $\tau_{\mathrm{c}}$ must be smaller than $300 \mathrm{Myr}$.

Models with intermediate coupling times, $\tau_{\mathrm{c}}=100$ and $200 \mathrm{Myr}$, reach a rotational state close to solid body rotation at the solar age. However, a ratio of $\Omega_{\text {core }} / \Omega_{\text {env }} \sim 2.5$ at $4.5 \mathrm{Gyr}$ obtained with $\tau_{\mathrm{c}}=200 \mathrm{Myr}$ should be regarded as an upper limit to plausible coupling times. Moreover, these intermediate models induce a behavior of the surface rotation opposite to observations.

Figure 2 shows the evolution of the envelope and core velocities for a coupling constant $\tau_{\mathrm{c}}=100 \mathrm{Myr}$. Before $1 \mathrm{Gyr}$, the radiative core maintains its maximal velocity and the gradual resurfacing of the angular momentum prevents the envelope 


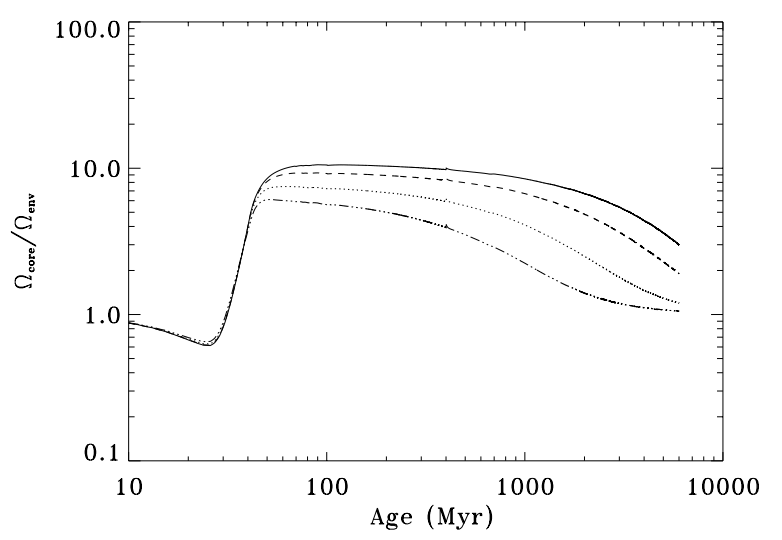

Fig. 1. $\Omega_{\text {core }} / \Omega_{\text {env }}$ from 10 to 6000 Myr for a $1 M_{\odot}$ star with solar composition. The solid line assumes a typical coupling time for the exchange of angular momentum between core and envelope of $\tau_{\mathrm{c}}=$ 300 Myr. The dashed, dotted and dot-dashed lines correspond to $\tau_{\mathrm{c}}=$ 200, 100, and $50 \mathrm{Myr}$, respectively.

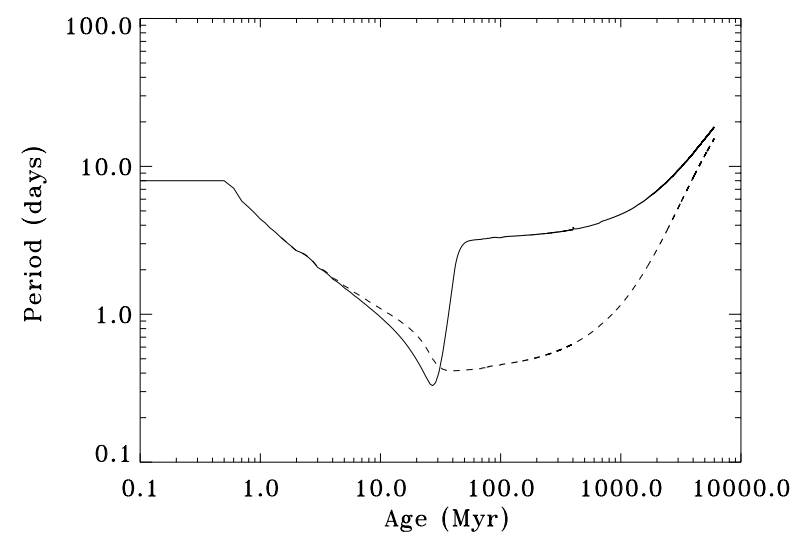

Fig. 2. Rotational evolution of the model with $\tau_{\mathrm{c}}=100 \mathrm{Myr}$. Solid and dashed lines are for the envelope and core, respectively. There is no significant evolution of the surface rotation rate from the Pleiades (100 Myr) to the Hyades (600 Myr), in disagreement with the observations.

from slowing down. This process occurs on the time scale $\tau_{\mathrm{c}}$ and there is little evolution of the equatorial surface speed between the Pleiades age and that of the Hyades. Such a pause in the surface rotational history is in disagreement with the observations of solar mass stars that show a smooth decrease of the rotation rate in the 0.9 to $1.1 M_{\odot}$ mass range during the early MS (e.g., Bouvier 1997).

An additional observational fact suggests that rotation must be rigid after a few $10^{8}$ years. The thinner convection zones of early G-type stars are more easily braked than those of early K-type stars. Thus, if we assume that decoupling between the core and the envelope still prevails after $600 \mathrm{Myr}$, then the Hyades rotation rates should not decrease from late-F to mid-K stars, as observed (Radick et al. 1987).

For all these reasons the model with $\tau_{\mathrm{c}}=50 \mathrm{Myr}$ seems a realistic one, yielding $\Omega_{\text {core }} / \Omega_{\text {env }} \simeq 2$ at 1 Gyr. Note that we would reach the same conclusion for smaller initial period and/or shorter lived circumstellar disk because of larger global angular momentum in the core. For small $\tau_{\mathrm{c}}$ the various instabilities induced by the vertical angular velocity gradient are far less probable than for larger $\tau_{c}$. Yet they could occur. For instance, as the specific angular momentum decreases near the radiation/convection boundary, the Rayleigh criterion for instability is met. Similarly, the secular shear instability is most likely present in this region as in the Sun today (Schatzman et al. 2000). However, we do not expect that a mixing process related to differential rotation with depth has a sufficient extent to affect the light elements. The reason lies on observations of the Hyades. Prior to this cluster age, solar-type stars experience very different rotational histories and one correspondingly expects a variety of differential rotation profiles with depth. If any of the associated processes were to affect light elements it would be difficult to explain why the Hyades exhibit such a small dispersion in the $T_{\text {eff }}-\log n(\mathrm{Li})$ relation. For the same reason, we also believe that deep mixing processes fueled by angular momentum loss are unlikely. We conclude that the mixing processes related to the vertical gradient of rotation or angular momentum loss probably do not play a role in history of light elements prior to the Hyades age. As these phenomena are most intense during the initial evolution, this strongly suggests that they do not affect directly or indirectly light elements along the entire MS. Finally, we note that the lack of a direct impact of angular momentum history on ${ }^{7} \mathrm{Li}$ is not in contradiction with the present observations of tidally locked binaries: the recent analysis of $\mathrm{BD}+22669$ and $\mathrm{BD}+23635$ in the Hyades cluster by Balachandran (2000) shows that these systems have the same value of ${ }^{7} \mathrm{Li}$ of single stars even though the angular momenta differ considerably.

\section{Constraints on the tachocline parameters}

In the previous section, we have summarized our model for the tachocline mixing and the prescriptions for the evolution of rotation on which the tachocline mixing depends. We have shown that it is likely that no significant differential rotation survives between the radiation and the convection zone after the Hyades age. Furthermore, the small spread in the $T_{\text {eff }}-\log n(\mathrm{Li})$ relation for the Hyades suggests that the process(es) responsible for the light elements history during MS neither rely on differential rotation with depth nor on angular momentum loss. The tachocline mixing complies with these requirements. Yet, as we have stressed before, this process depends on two critical parameters: the Brunt-Väisälä frequency and the tachocline thickness which we estimate below.

Since $\mathrm{Li}$ and Be burn at different temperatures, their coupled history provides stronger constraints on the internal mixing than just that of a single element. Thanks to the new measurements of ${ }^{9} \mathrm{Be}$ abundance in the late-F/early-G stars of M 67 and IC 4651 (Randich et al. 2002), we know that its surface abundance does not change appreciably during the MS phase up to at least the solar age. Thus, the mixing occurring at the top of the radiative zones required to explain the $\mathrm{Li}$-abundance pattern should not induce a deep circulation that would otherwise affect the beryllium fraction. For the solar case, BTZ have shown that beryllium is unaffected by tachocline mixing for plausible values of the parameters $N_{\mathrm{BV}}$ and $d_{\text {tacho. To investi- }}$ gate further the dependence of ${ }^{7} \mathrm{Li}$ and ${ }^{9} \mathrm{Be}$ depletion on these parameters and to extend the work of BTZ to other masses, 
we have calculated the evolution of stars with $1.04,1.00$ and $0.96 M_{\odot}$, varying both $N_{\mathrm{BV}}$ and $d_{\text {tacho }}$. As mentioned, our models were computed using the CESAM evolution code. We put particular care in following the changes of density and temperature at the base of the convective zone which is a critical quantity in determing the amount of light elements destruction.

We have started the models at the age of the Hyades and followed the evolution up to 6 Gyr. We have assumed an initial $\mathrm{Li}$ abundance of 2.5 dex for all models, corresponding to the mean value of 15 Hyades members with temperatures between 5600 and $6000 \mathrm{~K}$ (Thorburn et al. 1993). We mention that lithium abundances of solar-type stars in Praesepe, which is coeval to the Hyades, are in very good agreement with the latter: the mean $\mathrm{Li}$ abundances of Praesepe members in the same $T_{\text {eff }}$ range is $\log n(\mathrm{Li})=2.46$ (data from Soderblom et al. 1993), virtually the same as for the Hyades. In other words, one can safely assume that, whatever the initial conditions and the mixing mechanism before the Hyades age, by that time $\mathrm{Li}$ abundances of solar-type stars have converged to a unique value, independent on the particular cluster. Thus, the choice of our initial $\mathrm{Li}$ abundance appears solid and will not bias our results.

As mentioned before, the rotational tachocline mixing is induced by a time dependent rotation and latitude differential rotation. The rotation law follows the prescription given in Sect. 2.2 under solid body assumption $\left(\tau_{\mathrm{c}}=0 \mathrm{Myr}\right)$. This would be valid even if we had taken a value of $\tau_{\mathrm{c}}$ of $\sim 50 \mathrm{Myr}$. Then, the rotators follow the same slow-down regime which is only marginally different from Skumanich law (1972). We assumed that differential rotation scales with mean rotation as a power law $\tilde{\Omega} \propto \Omega^{n}$ with $n=0.7$, as empirically found by Donahue et al. (1996). This relation allows us to express the tachocline width as a function of rotational velocity as (see BTZ):

$d_{\text {tacho }} \propto \Omega^{(1.3 \pm 0.1) / 4}$.

We use this scaling law to follow the time variation of the tachocline thickness, calibrated to the value at the solar age as indicated by helioseismology. Note that significantly lower values of $n$ have been proposed: namely, $n=0.15$ (Hall 1991), and $n=-0.15$ (Kitchatinov \& Rüdiger 1999). However, the most recent observations of stars in the spectral range F0-G0 have found a value of $n=0.66 \pm 0.26$ (Reiners \& Schmitt 2003), in agreement with our choice. In any case, to investigate the dependence of the tachocline mixing on $n$, we have also run a model with $n=0(\tilde{\Omega}=$ const $)$.

Finally, since the structural changes near the base of the convection zone are extremely small between the age of the Hyades and the Sun, we expect that the buoyancy frequency in Eq. (1) does not vary with time; thus, we keep it constant in all the calculations.

\subsection{Results}

The results are reported in Tables 1 and 2, while in Fig. 3 our model predictions are compared to the observed distribution in M 67. In the upper panel, we consider models with a fixed tachocline thickness and with different values of $N_{\mathrm{BV}}$, while
Table 1. Depletion of ${ }^{7} \mathrm{Li}$ and ${ }^{9} \mathrm{Be}$ for $d_{\text {tacho }}=0.05 R_{\star}$ and varying $N_{\mathrm{BV}}$ at the solar age. The models start at the Hyades age with $\log n(\mathrm{Li})_{o}=2.5$ dex. The effective temperatures are $T_{\text {eff }}=5900 \mathrm{~K}$ for $1.04 M_{\odot}, T_{\text {eff }}=5780 \mathrm{~K}$ for $1.0 M_{\odot}$, and $T_{\text {eff }}=5640 \mathrm{~K}$ for $0.96 M_{\odot}$. The minimal ${ }^{9} \mathrm{Be}$ depletion of $\sim 0.9$ originates from microscopic diffusion.

\begin{tabular}{ccccccc}
\hline \hline$N_{\mathrm{BV}}$ & \multicolumn{2}{c}{$M_{*}=0.96 M_{\odot}$} & \multicolumn{2}{c}{$M_{*}=1.00 M_{\odot}$} & \multicolumn{2}{c}{$M_{*}=1.04 M_{\odot}$} \\
& $\mathrm{Li} / \mathrm{Li}$ & $\mathrm{Be} / \mathrm{Be} 。$ & $\mathrm{Li} / \mathrm{Li}$ & $\mathrm{Be} / \mathrm{Be} 。$ & $\mathrm{Li} / \mathrm{Li}$ 。 & $\mathrm{Be} / \mathrm{Be} 。$ \\
\hline 0.1 & $<10^{-10}$ & 0.61 & $6.7 \times 10^{-7}$ & 0.79 & $4.1 \times 10^{-3}$ & 0.91 \\
1 & $8.7 \times 10^{-6}$ & 0.83 & $4.4 \times 10^{-3}$ & 0.92 & 0.11 & 0.93 \\
10 & $5.3 \times 10^{-3}$ & 0.93 & 0.16 & 0.93 & 0.58 & 0.93 \\
100 & 0.10 & 0.93 & 0.61 & 0.94 & 0.80 & 0.92 \\
\hline
\end{tabular}

Table 2. Depletion of ${ }^{7} \mathrm{Li}$ and ${ }^{9} \mathrm{Be}$ for $N_{\mathrm{BV}}=10 \mu \mathrm{Hz}$ and varying $d_{\text {tacho }}$. Same values of $T_{\text {eff }}$ as in Table 1 .

\begin{tabular}{ccccccc}
\hline \hline$d_{\text {tacho }}$ & \multicolumn{2}{c}{$M_{*}=0.96 M_{\odot}$} & \multicolumn{2}{c}{$M_{*}=1.00 M_{\odot}$} & \multicolumn{2}{c}{$M_{*}=1.04 M_{\odot}$} \\
& $\mathrm{Li} / \mathrm{Li}_{\circ}$ & $\mathrm{Be} / \mathrm{Be}$ 。 & $\mathrm{Li} / \mathrm{Li}$ 。 & $\mathrm{Be} / \mathrm{Be}_{\circ}$ & $\mathrm{Li} / \mathrm{Li}$ 。 & $\mathrm{Be} / \mathrm{Be}$ 。 \\
\hline 0.05 & $5.3 \times 10^{-3}$ & 0.93 & 0.16 & 0.93 & 0.58 & 0.93 \\
0.1 & $1.4 \times 10^{-3}$ & 0.75 & $7.5 \times 10^{-3}$ & 0.88 & $5.7 \times 10^{-2}$ & 0.93 \\
0.2 & $1.7 \times 10^{-5}$ & 0.61 & $4.2 \times 10^{-3}$ & 0.58 & $3.1 \times 10^{-2}$ & 0.59 \\
\hline
\end{tabular}
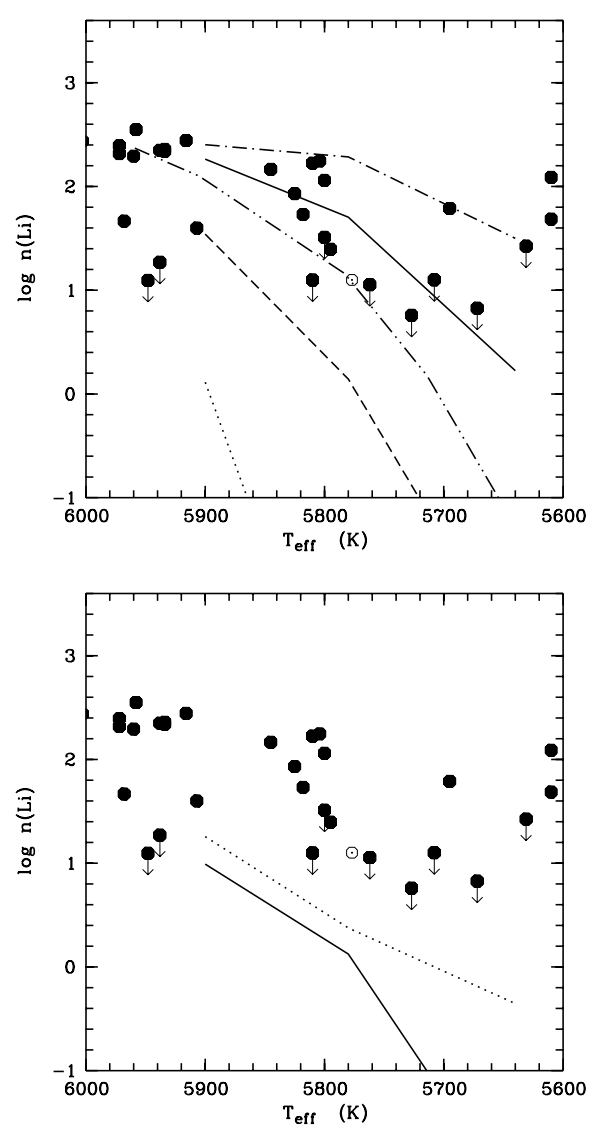

Fig. 3. Upper panel: model predictions with $d_{\text {tacho }}=0.05 R_{\star}$ and different values of $N_{\mathrm{BV}}$ compared to the observed distribution in M 67 (filled circles - data from Randich et al. 2002 and from Jones et al. 1999 reanalyzed by Randich et al. 2002). The solar value is also plotted. The dotted, dashed, dash-dot-dotted, solid and dash-dotted lines are for models with $N_{\mathrm{BV}}=0.1,1,2,10$ and $100 \mu \mathrm{Hz}$. Lower panel: comparison of the same data with models with $N_{\mathrm{BV}}=10 \mu \mathrm{Hz}$ and $d_{\text {tacho }}=0.2$ (solid curve) and $0.1 R_{\star}$ (dotted curve). 


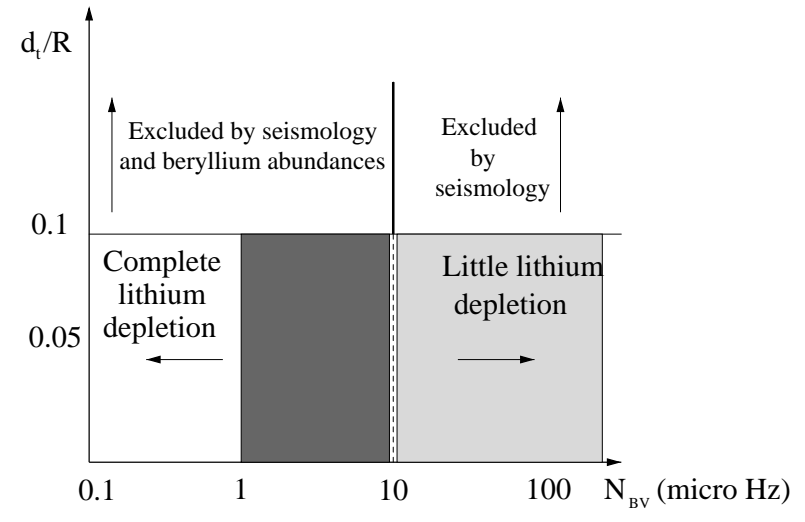

Fig. 4. Constraints on the tachocline mixing parameters. The shaded area corresponds to values of $d_{\text {tacho }}$ and $N_{\mathrm{BV}}$ frequency that match the requirements imposed by seismology and light elements abundances. Specifically, the black and light-grey areas correspond to the interval of frequencies that fit the lower and upper envelopes of M 67, respectively. The regions with no shading are excluded. Above $N_{\mathrm{BV}}=10 \mu \mathrm{Hz}$ the exclusion of thick tachocline region only relies on helioseismic measurements.

models with $N_{\mathrm{BV}}=10 \mu \mathrm{Hz}$ and two different values of $d_{\text {tacho }}$ are shown in the lower panel.

Figure 3 shows that models with $N_{\mathrm{BV}} \leq 1 \mu \mathrm{Hz}$ predict more Li depletion than observed and fail to fit even the lower envelope of the observed distribution. On the contrary, models with higher frequencies provide a better fit to the data; in particular the model with $N_{\mathrm{BV}}=100 \mu \mathrm{Hz}$ reproduces the upper envelope of the distribution, while in order to fit the lower envelope of the distribution and the Sun, acceptable values of $N_{\mathrm{BV}}$ are in the range $\sim 1$ to $10 \mu \mathrm{Hz}$. The models with $N_{\mathrm{BV}}$ in the range $1 \mu \mathrm{Hz}$ to $100 \mu \mathrm{Hz}$ predict very little Be depletion (less than $0.1 \mathrm{dex}$ ), in agreement with the observations.

Table 2 and the lower panel of Fig. 3 indicate that models with values $d_{\text {tacho }}$ of the tachocline depth larger than $0.05 R_{\star}$ and a Brunt-Väisälä frequency equal (or below) $10 \mu \mathrm{Hz}$ would not only produce too much $\mathrm{Li}$ depletion, but also destroy some $\mathrm{Be}$, in disagreement with the observations. Comparison of Tables 1 and 2 suggests that a combination of large values of $d_{\text {tacho }}$ and $N_{\mathrm{BV}}$ would result in the right amount of $\mathrm{Li}$ and (null) Be depletion. However, as mentioned in Sect. 2.1, values of $d_{\text {tacho }}>0.1 R_{\odot}\left(h_{\text {tacho }}>0.05 R_{\odot}\right)$ are in disagreement with seismic estimates of the solar tachocline. Note that for frequencies $N_{\mathrm{BV}} \leq 10 \mu \mathrm{Hz}$ large values of $d_{\text {tacho }}$ are also excluded for masses somewhat lower/higher than solar, implying that the tachocline thickness does not change significantly with mass, at least in the interval considered here.

Finally the amount of $\mathrm{Li}$ and Be destruction does not significantly vary in the model with $\tilde{\Omega}=$ const. For the $1 M_{\odot}$ star we find a variation of lithium depletion of $\sim 0.1$ dex and no change in beryllium, and similar values for the other masses.

Our conclusions on possible values of $N_{\mathrm{BV}}$ and $d_{\text {tacho }}$ are summarized in Fig. 4: the shaded area represents the region of the parameter space allowed by the constraints from light elements abundances and helioseismology. We see that while the tachocline thickness is rather well constrained, the buoyancy frequency is not. The latter can be better constrained using the Sun as a calibrator: specifically, if the Sun had had a surface lithium abundance of $2.5 \mathrm{dex}$ at the age of the Hyades, the value of $N_{\mathrm{BV}}$ that fits its present ${ }^{7} \mathrm{Li}$ abundance is $2 \mu \mathrm{Hz}$ (see Fig. 3). In the rest of the paper, we will use this value for the tachocline mixing, together with a depth of $d_{\text {tacho }}=0.05 R_{\star}$ at the solar age. Note however that we have assumed that the Sun is a typical star for its age and chemical composition; under the assumption that Li-rich stars in the upper envelope of M 67 and in the field are instead the standard and that the Sun is over-depleted in $\mathrm{Li}$, larger values of $N_{\mathrm{BV}}$ would be required.

\section{Metal abundances and mixing}

From the previous discussion we conclude that realistic values of the buoyancy frequency should lie between $\sim 1$ and $100 \mu \mathrm{Hz}$. Such a large interval could in principle account for the large dispersion in surface lithium abundance observed in M 67 . However, the possibility that otherwise similar stars are characterized by different values of $N_{\mathrm{BV}}$ is implausible and we believe that the reason for the dispersion must be a different one. In this section, we investigate whether and to what extent a small variation of the chemical composition could account for the observed $\log n(\mathrm{Li})$ spread.

Stars in open clusters are usually assumed to have the same chemical composition. However, a systematic study carried out by Nissen (1988) showed that a $[\mathrm{Fe} / \mathrm{H}]$ scatter of the order of \pm 0.1 dex (and up to \pm 0.15 dex) for Praesepe cannot be excluded. Similarly, the catalog of Cayrel de Strobel et al. (2001) suggests a small dispersion in the $[\mathrm{Fe} / \mathrm{H}]$ measurements of the Hyades and Pleiades. It is possible that variations of the same amplitude in $[\mathrm{O} / \mathrm{Fe}]$ or between other metals exist in these systems. Such changes can be expected because of the different sites of the nucleosynthesis of heavy elements.

\subsection{The effect of metals on the internal structure}

The extent of the outer convective region of MS stars is mainly sensitive to surface conditions, and depends weakly on metals at fixed effective temperature (Pinsonneault et al. 2001). This point is illustrated in Fig. 5 where we plot the quantity $2 \frac{T_{\mathrm{BCZ1}}-T_{\mathrm{BCZ2}}}{T_{\mathrm{BCZ} 1}+T_{\mathrm{BCZ} 2}}$ as a function of $T_{\mathrm{eff}} \cdot T_{\mathrm{BCZ1}}$ and $T_{\mathrm{BCZ} 2}$ are the temperatures at the base of the convection zone for two compositions: solar composition $(X=0.70821, Y=0.2722$, $Z=0.01959)$ and a mixture corresponding to the Hyades ( $X=0.7165, Y=0.26, Z=0.0235$ ). The figure shows that such a difference in composition induces differences of $T_{\mathrm{BCZ}}$ of less than $2 \%$ at a given $T_{\text {eff }}$.

Whatever the nature of the mixing process that induces lithium depletion on the MS, it is expected to become more efficient with increasing $T_{\mathrm{BCZ}}$. Metallicity variations change both $T_{\text {eff }}$ and $T_{\mathrm{BCZ}}$, but Fig. 5 shows that the $T_{\mathrm{eff}}-T_{\mathrm{BCZ}}$ relation remains mostly unaltered. No metallicity impact is therefore expected on the depletion pattern in the $T_{\text {eff }}-\log n(\mathrm{Li})$ plane, in agreement with the observations in young and middle-aged open clusters (Jeffries 1999; Jeffries \& James 1999).

At fixed effective temperature, the deviation of $T_{\mathrm{BCZ}}$ due to a metal fraction variation of $0.1 \mathrm{dex}$ is thus not large enough to produce the large $\log n(\mathrm{Li})$ scatter in M 67. This result holds 


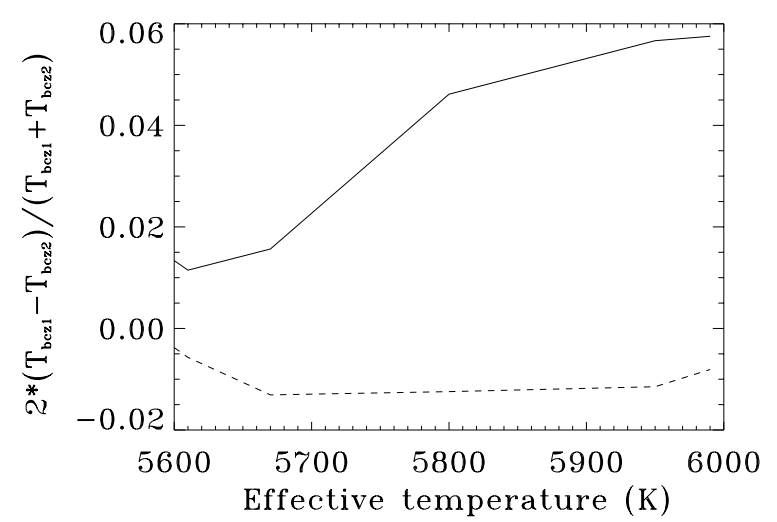

Fig. 5. Relation between the effective temperature and the temperature at the base of the convection zone. The dashed line corresponds to the ratio $2 \frac{T_{\mathrm{BCZ1}}-T_{\mathrm{BCZ2}}}{T_{\mathrm{BCZ1}}+T_{\mathrm{BCZ}}}$ for solar composition $(X=0.70821, Y=0.2722$, $Z=0.01959)$ and that of the Hyades $([\mathrm{Fe} / \mathrm{H}]=0.127 \mathrm{dex}, X=$ $0.7165, Y=0.26, Z=0.0235$ ). The solid line refers to the same ratio between solar composition and composition of Case 1: $X=0.7065$, $Y=0.2722, Z=0.0213$ but $[\mathrm{CNO} / \mathrm{Fe}]=+0.05$ dex. These relations have been computed for models at the age of the Hyades.

even if we consider the tachocline process at work simultaneously with convective mixing.

On the contrary, small variations of the fraction of specific elements can have a rather large impact on the internal structure (and on mixing processes). For example, the scatter in the $[\mathrm{Fe} / \mathrm{H}]$ abundance of cluster stars could imply a $[\mathrm{CNO} / \mathrm{Fe}]$ dispersion of similar amplitude (where we assume that carbon, nitrogen, and oxygen vary in a similar way). Using the Livermore web facilities ${ }^{1}$, we have computed opacity tables for non-solar metal ratios. In addition to the solar repartition, we have considered two cases in which CNO is increased and decreased by 0.05 dex relative to iron and the other metals (Cases 1 and 2, respectively).

Considering Case 1 , for a solar $[\mathrm{Fe} / \mathrm{H}]$ value the metal mix is richer in CNO than the Sun. This implies that in order to have the same effective temperature, the global metal mass fraction must be slightly increased. The reason is that the effective temperature is mainly fixed by bound-free transitions of $\mathrm{H}^{-}$ions in solar-like stellar atmospheres. The presence of these ions requires free electrons that between $5600 \mathrm{~K}$ and $6000 \mathrm{~K}$ are provided by metals at non negligible levels. Using the OPAL average ionization data, we compute that $\mathrm{Mg}, \mathrm{Fe}, \mathrm{Si}, \mathrm{Ca}$, and Al provide $43 \%$ of free electrons for typical solar atmospheric conditions with $T_{\text {eff }}=5800 \mathrm{~K}$ and $\rho=2.5 \times 10^{-7} \mathrm{~g} \mathrm{~cm}^{-3}$. Since in Case 1 the relative fractions of these metals in $\mathrm{Z}$ decreases as $\mathrm{CNO}$ increases, we must increase the total metal fraction by $10 \%$ to keep their total mass fraction constant. We also note that the iron fraction relative to hydrogen remains almost constant. On the one hand, we decrease the iron relative fraction among metals by increasing $\mathrm{CNO}$; on the other hand, we compensate the decrease by enhancing the global metal mass fraction. Thus, the new model will still maintain the same observables (effective temperature and $[\mathrm{Fe} / \mathrm{H}]$ ); however, for fixed thermodynamical conditions, the internal opacities will change due to their sensitivity to $\mathrm{CNO}$ abundances. As a result, both the extent of

\footnotetext{
${ }^{1}$ http://www-phys.1lnl.gov/Research/OPAL/index.html
}

Table 3. Effects of variations of the chemical compositions on stellar parameters. $Y=0.2722$ for all the cases considered here.

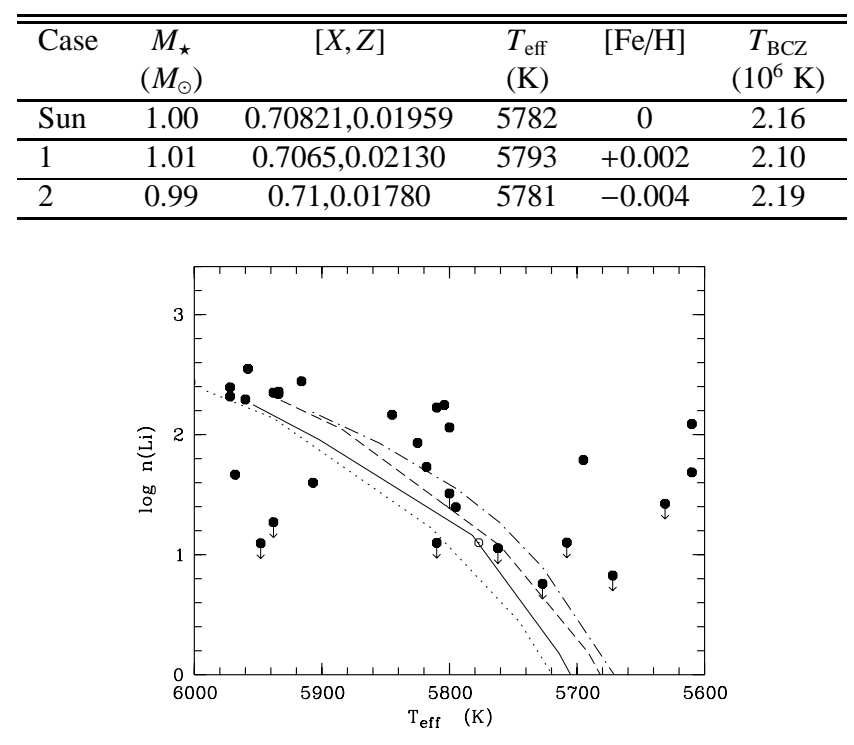

Fig. 6. The $\log n(\mathrm{Li}) \quad$ vs. $T_{\text {eff }}$ relation for solar-type stars. Observational data are the same as in Fig. 3. The solid, dashed and dotted lines correspond to the solar case and Cases 1 and 2, respectively. The dot-dashed line is for Case 1 with an helium mass fraction decreased by 0.005 (for this case we set $X=0.7115$ and $Y=0.2672$ to account for metal repartition and helium spread effects on the lithium history).

the convection zone and the temperature at its base will change. Figure 5 indeed shows that the change in the $T_{\mathrm{BCZ}}$ is larger than in the case of global metal variation. The results are summarized in Table 3 where we list the stellar parameters for the solar case and for different $[\mathrm{CNO} / \mathrm{Fe}]$ abundances.

\subsection{The lithium spread in M 67}

We have run our model with the tachocline mixing for three compositions (solar, Cases 1 and 2) and mass 0.96, 0.98, 0.99, $1.00,1.01,1.04$ and $1.06 M_{\odot}$. We have followed the evolution from the Hyades age up to $6 \mathrm{Gyr}$, using $N_{\mathrm{BV}}=2 \mu \mathrm{Hz}$ and $d_{\text {tacho }}=0.05 R_{\star}$.

The results are summarized in Fig. 6, where our model predictions at an age of $4.5 \mathrm{Gyr}$ for different chemical mixtures are again compared with the observed distribution of M 67.

Figure 6 shows that variations in chemical composition indeed result in different amounts of ${ }^{7} \mathrm{Li}$ depletion, with the CNO-rich (and globally metal rich) models (dashed curve) being the least lithium depleted. For the typical conditions near the bottom of the convection zone $(X=0.7, Y=0.28$, $\rho=0.5 \mathrm{~g} \mathrm{~cm}^{-3}$ and $\left.T=2.5 \times 10^{6} \mathrm{~K}\right)$ the OPAL opacity increases from $23.4 \mathrm{~cm}^{2} \mathrm{~g}^{-1}$ for Case 1 to $24.9 \mathrm{~cm}^{2} \mathrm{~g}^{-1}$ for Case 2. The reasons for this trend are not intuitive: CNO is the main opacity contributor in these conditions and yet when its fraction increases, the global opacity decreases. We have no simple explanation of this fact. A possible reason is that the derivative of opacity with respect to the iron fraction must be 
higher than that related to CNO (mainly dependent on oxygen) so that the decrease of iron and the accompanying increase of CNO eventually lower the overall opacity. Moreover we note the extreme sensitivity to changes of the relative abundances at temperatures where the bound-free edge for the $1 \mathrm{~s}$ level in hydrogenic nitrogen ion is very close to the maximum of the Rosseland mean weighting function.

The effect of chemical abundance variations on the lithium evolution is an encouraging result, although a small dispersion in metal abundances does not seem enough to entirely explain the observed ${ }^{7} \mathrm{Li}$ dispersion. At the effective temperature of the Sun, the difference between the CNO-rich and CNO-poor models is $\Delta \log n(\mathrm{Li})=0.48 \mathrm{dex}$. Although not negligible, this value is still below the observed scatter of 1-1.5 dex. To reach comparable levels, one would have to assume either a larger scatter in CNO abundances or a scatter at the Hyades age of $0.5-1$ dex, which is clearly above the observed value in this cluster (as well as in the even older case of IC 4651 - see Sect. 4.1).

Finally, we have to consider the effects of small variations of the helium abundance on the lithium spread. The results for a decrease of the He mass fraction of 0.005 dex are shown in Fig. 6, indicating a further reduction of the amount of $\mathrm{Li}$ destruction by about 0.2 dex with respect to Case 1 .

We end this section with two remarks. First, the scatter predicted by the models decreases significantly at higher than solar effective temperatures. This is due to the fact that the convective region becomes too shallow and the tachocline diffusion is no longer efficient. As shown in Fig. 6, this trend is not observed in M 67 where the spread is large even at $T_{\text {eff }}=6000 \mathrm{~K}$. However, this part of the diagram could include stars coming from the lithium dip: we suggest that some stars with $T_{\text {eff }}$ higher than $\sim 6000 \mathrm{~K}$ are indeed close to or at the end of the main sequence. For a $1.25 M_{\odot}$ mass star we predict a minimum value of $T_{\text {eff }}=6080 \mathrm{~K}$ at $3.7 \mathrm{Gyr}$, whereas it was $6410 \mathrm{~K}$ at $1 \mathrm{Gyr}$, corresponding to the red wing of the lithium dip. Given the age uncertainty of M $67\left(4_{0.5}^{+1} \mathrm{Gyr}\right.$, Demarque et al. 1992), stars with mass between $1.25 M_{\odot}$ and the turn-off value of $1.2 M_{\odot}$ would be difficult to distinguish from genuine MS stars just below the turn-off. Their luminosities are $\sim 1 \mathrm{mag}$ higher, but the stars with lithium measurements are among the brightest of the MS whose width in magnitude ( $\sim 1$ mag, Balachandran 1995) is comparable. Moreover the larger radii and masses give rise to similar surface gravities. Finally, we note that unlike G-type stars, those in the lithium dip do show beryllium depletion (Boesgaard \& King 2002). Conversely, the only possibly Be-depleted star (S988) in the Randich et al. (2002) sample of M 67 has $T_{\text {eff }}=6153 \mathrm{~K}$. We suggest that this star comes from the red wing of the lithium dip and that this could also be the case of the other most Li-depleted stars above $6000 \mathrm{~K}$.

The second point is that the average $\mathrm{Li}$ abundance of NGC 188 members with $T_{\text {eff }}=5800-6000 \mathrm{~K}$ is $\log n(\mathrm{Li}) \simeq$ $2.3 \pm 0.13$ dex (Randich et al. 2003); we also mention that NGC 188 does not show any significant star-to-star scatter. In other words, despite a very old age, NGC 188 does not exhibit a larger ${ }^{7} \mathrm{Li}$ depletion and dispersion than M 67, and is indeed more comparable to the younger stars of the Hyades. We are not able to reproduce the $\mathrm{Li}$ abundance of NGC 188, since we have calibrated our solar model using the $\mathrm{Li}$ abundance of the Sun (and of the lower envelope of M 67) which is a factor larger than 10 below the average of NGC 188 . However, on the one hand we note that the tachocline mixing beyond 4.5 Gyr is slow enough to explain the little (if any) $\mathrm{Li}$ depletion between the upper envelope of M 67 and NGC 188; on the other hand, we suggest that the detailed chemical composition and its possible variations between clusters could account for the difference between M 67 and NGC 188: for example, the very old cluster NGC 188 has formed at an epoch when the metal fractions and the associated scatter may have been different than in other more recent environments.

\section{Conclusion}

In this study, we have addressed three main issues regarding the evolution of the surface abundance of light elements in solar-type stars during the main sequence phase. First, we have derived constraints on the internal rotation and on the related dynamical phenomena after $\approx 1$ Gyr (Sect. 2 ). Second, we have explored the effects of changes in the parameters of the tachocline mixing, using the internal properties of the Sun as a calibrator. Thus, we have followed surface evolution of lithium and beryllium from the Hyades age up to 4.5 Gyr and compared the results to the observations of M 67 (Sect. 3). Third, we have looked at the effect of variations of the detailed chemical composition in stars of mass between 0.96 and $1.04 M_{\odot}$, and have investigated the coupling between opacity and the tachocline mixing (Sect. 4). Our main results can be summarized as follows:

i) In agreement with other studies that have claimed short coupling times, we conclude that the characteristic time for angular momentum transport between core and envelope cannot exceed $\sim 50$ Myr. This suggests that solar-type stars do not maintain significant differential rotation with depth after $\sim 1$ Gyr. In turn, this restricts the range of possible mixing processes needed to explain the subsequent surface evolution of lithium and beryllium. In particular, since rotation is slow and almost solid after $\sim 1$ Gyr, processes associated with shear and angular momentum loss likely have a small impact on the late MS history of light elements.

ii) The tachocline process (Spiegel \& Zahn 1992) is neither related to differential rotation with depth nor to angular momentum internal transfer and appears very appealing to help explaining the light elements evolution. This mechanism is able to reproduce both lithium and beryllium histories for plausible values of its parameters. The tachocline width parameter is constrained by helioseismology that suggests that it does not exceed $0.05 R_{\odot}$. Thanks to lithium and beryllium abundances we can also put limits on this quantity for masses slightly larger/smaller than solar. The value of the buoyancy frequency that reproduces the solar lithium abundance is $2 \mu \mathrm{Hz}$. A larger buoyancy frequency is needed to reproduce the upper envelope of M 67. We stress that for a large range of buoyancy frequency values the tachocline mixing does not induce any beryllium depletion, in agreement with the observations. 
iii) Using the calibration on the Sun, we have followed the evolution of stars with mass between 0.96 and $1.04 M_{\odot}$ and different chemical composition. We suggest that a difference in the $[\mathrm{CNO} / \mathrm{Fe}]$ ratio of 0.1 dex can change the temperature at the base of the convection zone by $\sim 9 \times 10^{4} \mathrm{~K}$, at fixed effective temperature and $[\mathrm{Fe} / \mathrm{H}]$ abundance; such a difference is large enough to lead to a $\log n(\mathrm{Li})$ scatter of $\approx 0.5$ dex at the age of M 67. Although this is smaller than the observed spread in $M$ 67, our results are very encouraging and show that the star-to-star scatter in lithium in M 67 could be explained by a star-to-star scatter in heavy element abundances.

In conclusion, the history of rotation, ${ }^{7} \mathrm{Li}$ and ${ }^{9} \mathrm{Be}$ abundances coupled with the results of helioseismology allows us to derive some constraints on the dynamical internal processes of solar-like stars, such as the tachocline diffusion. This mechanism rests upon rotation and differential rotation with latitude. At present, the variation of differential rotation with depth is not directly accessible by measurements, but asteroseismical experiments in space could change this situation in the near future. The way surface differential rotation in latitude evolves with global rotation is in principle an important ingredient. However, differences in the estimates of the dependence of $\tilde{\Omega}$ on $\Omega$ do not seem to have a significant impact on our predictions of Li and Be depletion. Equally important is the precise determination of the opacity in the regions close to the lower boundary of convection. For this reason, a refinement of the mesh grid of the opacity tables as function of temperature and density for the thermodynamical conditions appropriate to these critical regions is clearly needed. From the observational viewpoint, we stress the relevance of accurate measurements of specific elements, in particular oxygen. Such studies would shed light on the impact of subtle chemical composition effects on light element abundances that should be taken into account in more refined theoretical models.

Acknowledgements. This research was carried out with the support from the Italian MIUR COFIN 2000 grant and from the French Ministry for foreign affairs Lavoisier Grant.

\section{References}

Anders, E., \& Grevesse, N. 1989, Geochim. Cosmochim. Acta., 53, 197

Attridge, J. M., \& Herbst, W. 1992, ApJ, 398, L61

Balachandran, S. 1995, ApJ, 446, 203

Balachandran, S. 2000, IAUJD 5, to appear in Highlights of Astronomy, vol. 12, ed. H. Rickman (Kluwer)

Barnes, G., Charbonneau, P., \& MacGregor, K. B. 1999, ApJ, 511, 466

Boesgaard, A. M., \& Budge, K. G. 1989, ApJ, 338, 875

Boesgaard, A. M., \& King, J. R. 2002, ApJ, 565, 587

Bouvier, J. 1997, Mem. SAIt, 68, 881

Bouvier, J., Forestini, M., \& Allain, S. 1997, A\&A, 326, 1023

Brun, A. S., Turck-Chièze, S., \& Zahn, J. P. 1999, ApJ, 525, 1032 (BTZ)

Cayrel de Strobel, G., Soubiran, C., \& Ralite, N. 2001, A\&A, 373, 159

Chaboyer, B., Demarque, P., \& Pinsonneault, M. H. 1995, ApJ, 441, 865

Charbonneau, P., Christensen-Dalsgaard, J., Henning, R., et al. 1999, ApJ, 527, 445
Choi, P. I., \& Herbst, W. 1996, AJ, 111, 283

Corbard, T., Berthomieu, G., Morel, P., et al. 1997, A\&A, 324, 298

Corbard, T., Blanc-Feraud, L., Berthomieu, G., \& Provost, J. 1999, A\&A, 344, 696

Corbard, T., Jiménez-Reyes, S. J., Tomczyk, S., Dikpati, M., \& Gilman, P. 2001, Proceedings of the SOHO 10/GONG 2000 Workshop: Helio- and asteroseismology at the dawn of the millennium, 2-6 October 2000, ed. A. Wilson, \& P. L. Palle, ESA SP-464, 265

Deliyannis, C. P. 2000, ASP Conf. Ser., 198, 225

Deliyannis, C. P., \& Pinsonneault, M. 1997, ApJ, 488, 836

Demarque, P., Greene, E. M., \& Guenther, D. B. 1992, AJ, 103, 151

Donahue, R. A., Saar, S. H., \& Baliunas, S. L. 1996, ApJ, 366, 484

Elliott, J. R., \& Gough, D. O. 1999, ApJ, 516, 475

García López, R. J., Rebolo, R., \& Perez de Taoro, M. R. 1995, A\&A, 302, 184

Hall, D. S. 1991, in Learning about the stellar dynamos from longterm photometry of starspots, ed. I. Tuominen, D. Moss, G. \& Ruediger, IAU Coll. 130, The Sun and Cool Stars: Activity, Magnetism, Dynamos. (Berlin: Springer), 153

Herbst, W., Rhode, K. L., Hillenbrand, L. A., \& Curran, G. 2000, AJ, 119, 261

Jeffries, R. D. 1999, MNRAS, 304, 821

Jeffries, R. D. 2000, ASP Conf. Ser., 198, 245

Jeffries, R. D., \& James, D. J. 1999, ApJ, 511, 218

Jones, B. F., Fisher, D., \& Soderblom, D. R. 1999, AJ, 117, 330

Kawaler, S. D. 1988, ApJ, 333, 236

Kitchatinov, L. L., \& Rüdiger, G. 1999, A\&A, 344, 911

Krishnamurthi, A., Pinsonneault, M. H., Barnes, S., \& Sofia, S. 1997, ApJ, 480, 303

MacGregor, K. B., \& Brenner, M. 1991, ApJ, 376, 204

Martìn, E. L., \& Montes, D. 1997, A\&A, 318, 805

Montalbán, J., \& Schatzmann, E. 2000, A\&A, 354, 943

Morel, P 1997, A\&AS, 124, 597

Nissen, P. E. 1988, A\&A, 199, 146

Pasquini, L. 2000, in The Light Elements and their Evolution, ed. L. da Silva, M. Spite, \& J. R. de Medeiros, IAU Symp., 198, 245

Pasquini, L., Liu, Q., \& Pallavicini, R. 1994, A\&A, 287, 191

Piau, L., \& Turck-Chièze, S. 2002, ApJ, 566, 419

Pinsonneault, M. H., Kawaler, S. D., Sofia, S., \& Demarque, P. 1989, ApJ, 338, 424

Pinsonneault, M. H., DePoy, D. L., \& Coffee, M. 2001, ApJ, 556, L59

Radick, R. R., Thompson, D. T., Lockwood, G. W., Duncan, D. K., \& Baggett, W. E. 1987, ApJ, 321, 459

Randich, S., Pasquini, L., \& Pallavicini, R. 2000, A\&A, 356, L25

Randich, S., Pallavicini, R., Meola, G., Stauffer, J., \& Balachandran, S. C. 2001, A\&A, 372, 862

Randich, S., Primas, F., Pasquini, L., \& Pallavicini, R. 2002, A\&A, 387, 222

Randich, S., Sestito, P., \& Pallavicini, R. 2003, A\&A, 399, 133

Reiners, A., \& Schmitt, J. H. M. M. 2003, A\&A, 398, 647

Rüdiger, G., \& Brandenburg, A. 1995, A\&A, 296, 557

Schatzman, E., Zahn, J.-P., \& Morel, P. 2000, A\&A, 364, 876

Schou, J., Howe, R., Basu, S., et al. 2002, ApJ, 567,1234

Skumanich, A. 1972, ApJ, 171, 565

Soderblom, D. R., Fedele, S. B., Jones, B. F., Stauffer, J. R., \& Prosser, C. F. 1993, AJ, 106, 1080

Spiegel, E. A., \& Zahn, J. P. 1992, A\&A, 265, 106

Swenson, F. J., \& Faulkner, J. 1992, ApJ, 395, 654

Talon, S., Kumar, P., \& Zahn, J.-P. 2002, ApJ, 574, 175

Thorburn, J. A., Hobbs, L. M., Deliyannis, C. P., \& Pinsonneault, M. H. 1993, ApJ, 415, 150

Zahn, J.-P., Talon, S., \& Matias, J. 1997, A\&A, 322, 320 